\title{
Agregação e matéria orgânica de um argissolo sob diferentes preparos do solo para plantio de Eucalipto
}

\author{
Juliana Prevedello', Eduardo Saldanha Vogelmann', Douglas Rodrigo Kaiser ${ }^{2}$, Eracilda Fontanela ${ }^{3}$, Dalvan José Reinert', \\ José Miguel Reichert ${ }^{1}$ \\ ${ }^{1}$ Universidade Federal de Santa Maria, Departamento de Solos, Av. Roraima, 1000, Camobi, CEP 97105900, Santa Maria, RS, Brasil \\ ${ }^{2}$ Universidade Federal da Fronteira Sul, Rua Major Antônio Cardoso, 590, Centro, CEP 97900-000, Cerro Largo, RS, Brasil \\ ${ }^{3}$ Universidade Federal do Pampa, Campus Alegrete, Av. Tiaraju, 810, Ibirapuitã, CEP 97546550, Alegrete, RS, Brasil
}

\author{
"Autor correspondente: \\ juliprevedello@gmail.com \\ Termos para indexação: \\ Manejo do solo \\ Estabilidade de agregados \\ Estrutura do solo \\ Eucalyptus grandis
}

Index terms:

Soil management

Aggregate stability

Soil structure

Eucalyptus grandis

Histórico do artigo:

Recebido em 30/11/2012

Aprovado em 11/06/2014

Publicado em 07/07/2014

doi: 10.4336/2014.pfb.34.78.456
Resumo - A agregação pode sofrer alterações permanentes ou temporárias com variações cíclicas provocadas por práticas de manejo do solo e pelo crescimento das plantas. O estudo avaliou a variação temporal e espacial da estabilidade de agregados estáveis em água e a sua relação com o conteúdo de matéria orgânica em um Argissolo em Santa Maria, RS, Brasil. Os tratamentos avaliados foram quatro sistemas de preparo do solo para implantação de povoamento de Eucalyptus grandis: plantio direto; escarificação; escarificação seguida de gradagem e enxada rotativa. Amostras de solo foram coletadas na camada de 0,00-0,05 m para a determinação da densidade, da estabilidade dos agregados estáveis em água e do teor de matéria orgânica do solo. A escarificação, escarificação seguida de gradagem e enxada rotativa resultaram em menor estabilidade estrutural de agregados estáveis em água. Os efeitos negativos do preparo do solo sobre o Argissolo de textura franco arenosa são reduzidos após 12 meses, demonstrando sua capacidade em reestabelecer a estrutura e a agregação. Os diferentes preparos do solo avaliados não foram capazes de aumentar o teor de carbono orgânico, não influenciado no estado de agregação. O crescimento inicial do eucalipto foi favorecido com a intensificação do preparo do solo.

\section{Aggregation and organic matter under alfisol soil tillage for Eucalyptus plantation}

\begin{abstract}
Aggregation can suffer permanent or temporary changes to cyclical variations induced by management practices for soil and plant growth. The study evaluated the temporal and spatial variation of the stability of water stable aggregates and its relationship with the organic matter content in an Hapludalf in Santa Maria municipality, Rio Grande do Sul State, Brazil. The treatments were four tillage systems for deployment of Eucalyptus grandis: no-tillage, chisel, chisel tillage plus harrowing and rotary tiller. Soil samples were collected in the layer of 0.00 to $0.05 \mathrm{~m}$ for determination of the density, and stability in water of stable organic matter aggregates contents of the soil. Chisel, chisel tillage plus harrowing and rotary tiller resulted in lower structural stability of water stable aggregates. The negative effects of tillage on Hapludalf with sandy loam texture are reduced after 12 months, demonstrating its ability to restore the structure and aggregation. The soil tillage evaluated did not increase the carbon content and that was not influenced by the state of aggregation. The early growth of Eucalyptus was favored by the intensification of soil tillage.
\end{abstract}




\section{Introdução}

A estrutura do solo é representada pela agregação e tem sido definida como sendo o resultado da interação entre o tamanho, forma e arranjo das partículas sólidas e espaços porosos do solo, sendo altamente variável e associado aos fatores físicos, químicos e biológicos (Letey, 1991). O acúmulo de matéria orgânica do solo, que pode ser obtido pelo manejo adequado e pelos resíduos culturais acumulados na superfície, geralmente está associado a uma melhoria das condições físicas (Reichert et al., 2011), especialmente relacionado aos seus benefícios à estrutura (Baumgartl \& Horn, 1991; Reichert et al., 2009). A variação na quantidade de matéria orgânica do solo (MOS) é dependente da espécie e de seus resíduos acumulados na superfície, além de fatores como a profundidade do solo, clima, idade das árvores, práticas silviculturais, textura e mineralogia do solo (Zinn et al., 2007; 2011). As espécies florestais contribuem positivamente para a formação e estabilização de agregados, tanto em função do sistema radicular denso e bem distribuído no perfil do solo quanto ao elevado aporte de MOS (Bernoux et al., 2009; Yang et al., 2009).

O tamanho dos agregados e o estado de agregação do solo podem ser determinados de várias formas. Segundo Kemper \& Chepil (1965) podem ser usados como parâmetros o diâmetro médio ponderado (DMP), o diâmetro médio geométrico (DMG) e o índice de estabilidade de agregados (IEA). Cada um deles apresenta um princípio diferente: o DMP é tanto maior quanto maior for a percentagem de agregados grandes retidos nas peneiras com malhas maiores; o DMG representa uma estimativa do tamanho da classe de agregados de maior ocorrência e o IEA representa uma medida da agregação total do solo e não considera a distribuição por classes de agregados.

A interação entre a matéria orgânica do solo, a textura e a mineralogia definem a estabilidade e o tamanho dos agregados (Zinn et al., 2007). Conforme modelo proposto por Tisdall \& Oades (1982), a estrutura foi baseada em macro e microagregados e partículas livres no solo. Os microagregados representam as unidades básicas e possuem diâmetro menor que $0,250 \mathrm{~mm}$, sendo compostos principalmente por argilas, complexos de matéria orgânica, além de cátions polivalentes ( $\mathrm{Fe}, \mathrm{Al}$ e $\mathrm{Ca}$ ) (Edwards \& Bremner, 1967; Tisdall \& Oades, 1982). Por sua vez, solos bem estruturados e estabilizados possuem grande quantidade de macroagregados, compostos por microagregados unidos por uma rede de hifas de fungos e raízes. Dessa forma, a matéria orgânica do solo apresenta-se protegida no interior dos agregados em solos não perturbados. Entretanto, o cultivo do solo promove a liberação da matéria orgânica, tornando-a vulnerável à decomposição, aumentando a susceptibilidade aos processos erosivos (Tisdall \& Oades, 1982).

No sistema plantio direto, a inexistência de revolvimento do solo associada à manutenção de uma camada de resíduos orgânicos na superfície, permite um enriquecimento de material orgânico, com prováveis reflexos na estrutura e na resistência do solo à compactação (Reichert et al., 2011). Isso foi verificado por Kumaria et al. (2011) e Franchini et al. (2012), que encontraram elevados índices de agregação sob plantio direto em relação ao sistema convencional, e justificaram esse efeito pelos altos teores de carbono orgânico e atividade microbiana no solo. No entanto, Kumaria et al. (2011) e Zinn et al. (2011) verificaram que o diâmetro médio geométrico dos agregados foi pouco influenciado pelo teor de carbono orgânico.

O teor de matéria orgânica possui relação direta com o manejo e com as culturas empregadas. Beldini et al. (2010) encontraram diâmetro médio geométrico de agregados em mata nativa superior aos de povoamentos de Eucalyptus sp. Segundo os autores, a mata nativa possui maior grau de estruturação do solo, devido à diversidade de espécies vegetais com diferentes sistemas radiculares, além do grande aporte de serapilheira na superfície, que favorecem a atividade dos organismos e, consequentemente, a estabilidade de agregados do solo.

O sul do Brasil tem se mostrado promissor à expansão da silvicultura, como uma área de interesse para a implantação de novos plantios de eucalipto, sobretudo na metade sul, devido à rusticidade e adaptabilidade da espécie às diferentes condições edafoclimáticas, além de constituir matéria-prima para a produção de diversos produtos como celulose, carvão, lenha, madeira serrada, entre outros. No entanto, os processos físicos relacionados com a implantação e tráfego de máquinas agrícolas e florestais, clima e crescimento de plantas podem causar alterações na estrutura do solo ao longo do tempo e em função do local da avaliação. Tais variações são chamadas de temporais e espaciais e podem ocorrer em um curto ou longo espaço de tempo (Saha et al., 2010).

Na literatura constam estudos envolvendo a variação da estabilidade estrutural em áreas agrícolas (Pikul 
Junior et al., 2009; Saha et al., 2010; Kumaria et al., 2011; Franchini et al., 2012), porém, em áreas de florestas os estudos ainda são incipientes. Dessa forma, o presente estudo teve por objetivo avaliar a variação temporal e espacial da estabilidade de agregados estáveis em água e a sua relação com o conteúdo de matéria orgânica em um Argissolo Vermelho-Amarelo submetido a quatro sistemas de preparo do solo para implantação de povoamento de Eucalyptus grandis.

\section{Material e métodos}

O estudo foi conduzido em uma área experimental pertencente à Fundação Estadual de Pesquisa Agropecuária, no Município de Santa Maria, Rio Grande do Sul, Brasil. O clima da região é classificado como subtropical úmido (Cfa), segundo a classificação de Köppen. A temperatura e precipitação média anual são de $19^{\circ} \mathrm{C}$ e $1.769 \mathrm{~mm}$, respectivamente (Moreno, 1961). O solo foi classificado, segundo o Sistema Brasileiro de Classificação do Solo, como Argissolo VermelhoAmarelo Distrófico arênico (Santos et al., 2006) e possui valores médios de $165 \mathrm{~g} \mathrm{~kg}^{-1}$ de argila, $195 \mathrm{~g} \mathrm{~kg}^{-1}$ de silte e $640 \mathrm{~g} \mathrm{~kg}^{-1}$ de areia (classe textural franco arenosa) na camada de $0,00-0,30 \mathrm{~m}$. Antes da instalação do experimento, a área encontrava-se em pousio por mais de 20 anos e a vegetação era composta principalmente por gramíneas, sem apresentar características de degradação.

O delineamento experimental utilizado foi o de blocos ao acaso com 3 repetições e os tratamentos avaliados foram quatro sistemas de preparo do solo para implantação de povoamento de Eucalyptus grandis Hill ex Maiden.: sem preparo ou plantio direto (PD); escarificador até $0,30 \mathrm{~m}$ de profundidade (Esc); escarificador até $0,30 \mathrm{~m}$ de profundidade seguida de grade niveladora (EG) e enxada rotativa até $0,20 \mathrm{~m}$ de profundidade (ER), em parcelas de $20 \mathrm{~m}$ x $30 \mathrm{~m}$.

O plantio direto caracterizou-se por receber coveamento manual com auxílio de um trado, sendo posteriormente aplicado o herbicida glifosato, na dosagem de $720 \mathrm{~g} \mathrm{ha}^{-1}$, em uma faixa de $1 \mathrm{~m}$ de largura, na linha de plantio. Nesse tratamento, as mudas foram transplantadas em covas de $0,10 \mathrm{~m}$ de profundidade e $0,08 \mathrm{~m}$ de diâmetro. Para a implantação dos demais tratamentos, foi utilizado um trator, sendo a escarificação realizada com um escarificador de 1 haste inclinada até uma profundidade de $0,30 \mathrm{~m}$, espaçadas a cada $3,0 \mathrm{~m}$. No tratamento escarificado seguido de gradagem, a escarificação foi seguida por uma passagem de grade de disco "off-set" ou em "V" com uma profundidade de operação de $0,10 \mathrm{~m}$. O preparo com enxada rotativa mobilizou uma faixa de $1,0 \mathrm{~m}$ de largura e $0,20 \mathrm{~m}$ de profundidade. As mudas de $E$. grandis foram plantadas no dia 28 de novembro de 2006, em espaçamento de 2,0 m x 3,0 m, sendo cada parcela composta por 100 plantas, distribuídas em 10 linhas de 10 plantas.

Amostras de solo indeformadas foram coletadas em cilindros metálicos de $0,06 \mathrm{~m}$ de diâmetro e $0,05 \mathrm{~m}$ de altura, na camada de 0,00-0,05 m para a determinação da densidade do solo, segundo metodologia descrita em Claessen (1997). Na mesma camada, coletaram-se amostras de solo com estrutura preservada, na linha e entrelinha de plantio, com o auxílio de uma pá de corte, para a avaliação da agregação. As coletas foram realizadas em três épocas distintas: antes da aplicação dos tratamentos (época 1), com o objetivo de caracterizar a área experimental; três meses após o preparo do solo (época 2) e doze meses após o preparo do solo (época 3).

A separação e a estabilidade dos agregados em água foram determinadas em amostras de agregados menor que $8 \mathrm{~mm}$, conforme método modificado de Kemper $\&$ Chepil (1965), com peneiras de malha de 4,76 mm; 2,00 mm; 1,00 mm e 0,25 mm de diâmetro. Neste método, os agregados foram separados manualmente, observando-se as superfícies naturais de clivagem e, posteriormente, umedecidos por capilaridade por $10 \mathrm{~min}$, agitados por $10 \mathrm{~min}$, com 30 oscilações $\mathrm{min}^{-1}$ e curso de oscilação vertical de 0,038 m (Yoder, 1936).

Aestabilidade dos agregados estáveis em água foi expressa pelo diâmetro médio geométrico (DMG), diâmetro médio ponderado (DMP) e percentagem de agregados (AGRi) por classe de diâmetro $(8,00-4,76 \mathrm{~mm} ; 4,76-2,00 \mathrm{~mm} ; 2,00$ $1,00 \mathrm{~mm} ; 1,00-0,25 \mathrm{~mm}$ e $<0,25 \mathrm{~mm})$. As equações utilizadas para os cálculos seguiram as proposições da metodologia de Kemper \& Chepil (1965):

$$
\begin{aligned}
& \mathrm{DMG}=\operatorname{EXP}\left(\Sigma\left(A G R_{i} \times \ln \left(d_{i}\right) / \Sigma A G R_{i}\right)\right) \\
& \mathrm{DMP}=\Sigma\left(d_{i} \times A G R_{i}\right) \\
& A G R_{i}(\%)=\left(m A G R_{i}\right) /\left(\Sigma A G R_{i}\right)
\end{aligned}
$$

Em que: $m A G R_{i}=$ massa de agregados da classe i (menos areia); $\Sigma A G R_{i}=$ massa total de agregados (menos areia); $\ln =$ logaritmo neperiano; $d i=$ diâmetro médio de classe i; $A G R i$ $(\%)=$ percentagem de agregados por classe de tamanho.

O teor de carbono orgânico do solo foi determinado pelo método de combustão úmida, modificado e descrito 
por Tedesco et al. (1995). Os valores de carbono orgânico do solo obtidos foram convertidos em matéria orgânica do solo multiplicando-se o primeiro por 1,724 , pois admitiu-se que o carbono orgânico do solo participa com 58\% na composição da matéria orgânica do solo (Claessen, 1997).

Os resultados obtidos foram submetidos à análise de variância e, quando o valor do teste $\mathrm{t}$ foi significativo, utilizou-se o teste de Tukey ao nível de 5\% de significância para a comparação das médias dos tratamentos. Para análise dos dados de diâmetro médio geométrico, diâmetro médio ponderado, matéria orgânica do solo e percentagem de agregados por classe de tamanho nas diferentes épocas de coleta (variação temporal), considerou-se o sistema de manejo como fator principal e a época de avaliação como fator secundário, constituindo-se um modelo de análise com parcela subdividida. Para diferentes locais de coleta (variação espacial), considerou-se o sistema de manejo como fator principal e o local de coleta como fator secundário. Além disso, foram realizadas análises de correlação de Pearson entre as propriedades físicas.

\section{Resultados e discussão}

A distribuição do tamanho de agregados estáveis em água para as diferentes épocas avaliadas encontram-se na Figura 1. Os tratamentos não apresentaram diferença estatística entre si, porém verifica-se variação entre as épocas de coleta, ou seja, variação temporal (Tabela 1). A maior concentração de agregados foi encontrada antes dos preparos do solo (época 1) na classe de maior diâmetro $(8,00-4,76 \mathrm{~mm})$, variando de $40 \%$ a $50 \%$, com aumento nas classes de menor diâmetro $(1,00$ $0,25 \mathrm{~mm}$ e $<0,25 \mathrm{~mm}$ ) após o revolvimento (época 2), demonstrando um reflexo negativo da mobilização do solo sobre a estabilidade de agregados, corroborando com o observado em áreas agrícolas por Castro Filho et al. (1998), Fuentes et al. (2009) e Kumaria et al. (2011), e florestais por Beldini et al. (2010) e Zinn et al. (2011). Conforme relatam Zinn et al. (2011), a quebra dos agregados de maior diâmetro é esperada após o uso do solo, em função da sua maior porosidade, baixa tensão à força de desagregação e numerosos planos de fratura, com consequente aumento nas classes de menor diâmetro.

Após 12 meses do preparo (época 3), o solo demonstrou recuperação na sua agregação, evidenciada pelos valores de distribuição de agregados da classe de maior diâmetro, semelhante ao encontrado nos tratamentos antes da mobilização do solo (época 1). A interação do crescimento e desenvolvimento das árvores e dos demais fatores físicos, químicos e biológicos atuam conjuntamente no processo de agregação e favorecem a recuperação da estrutura do solo, além do que, solos arenosos possuem a capacidade de recuperar sua estrutura mais rapidamente que os argilosos (Yang et al., 2009; Reichert et al., 2009; Tang et al., 2011). Em solos arenosos, as partículas de quartzo são impregnadas por partículas finas de argila e coloides de carbono orgânico do solo (Zinn et al., 2007), que são mais susceptíveis ao ataque microbiano do que solos de textura mais fina, como os argilosos e siltosos (Zinn et al., 2011).

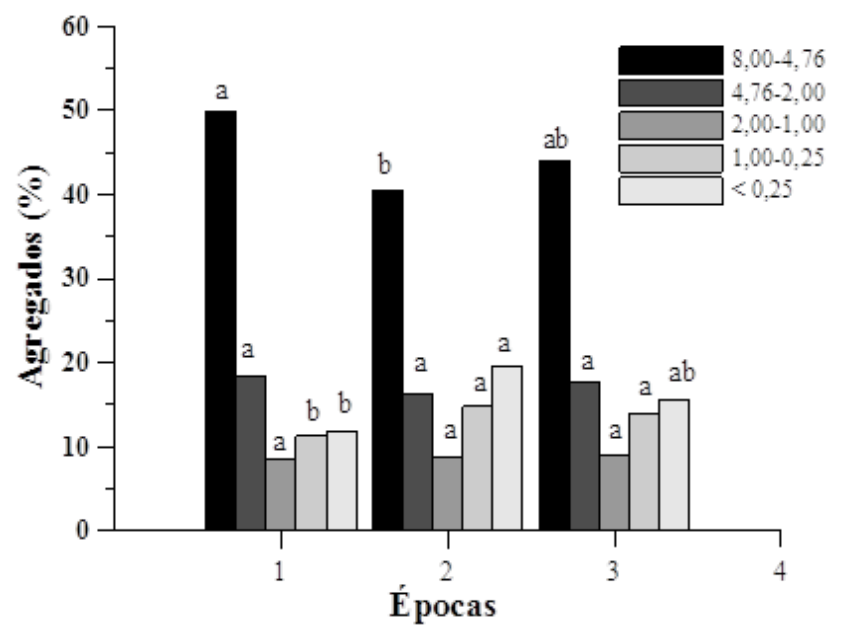

Figura 1. Distribuição do tamanho de agregados estáveis em água (menor que $8 \mathrm{~mm}$ ) em Argissolo nas diferentes épocas de avaliação. Valores seguidos de mesma letra entre as épocas, não diferem estatisticamente pelo teste de Tukey a $5 \%$ de probabilidade.

Os diferentes preparos do solo e épocas de avaliação apresentaram interação significativa para a densidade (Tabela 2). A maior densidade foi verificada no solo sem preparo, denominado de plantio direto, quando comparado com os tratamentos com revolvimento mínimo, com diferença significativa nas épocas 2 e 3 . Gonçalves (2002) argumenta que preparos do solo menos intensivos possuem ação semelhante ao plantio direto. Entretanto, promovem a descompactação por reduzir a densidade e a resistência do solo à penetração das raízes das plantas (Reichert et al., 2009), além de favorecer a liberação de nutrientes de forma lenta e gradual, por meio da mineralização, o que facilita o estabelecimento e o crescimento inicial das mudas de eucalipto. 
Tabela 1. Valores de $\mathrm{F}$ e coeficiente de variação (CV \%) para valores de densidade do solo (Ds), diâmetro médio geométrico (DMG), diâmetro médio ponderado (DMP) e teor de matéria orgânica do solo (MOS\%) dos agregados de tamanho menor que $8 \mathrm{~mm}$ na camada $0,00-0,05 \mathrm{~m}$, nos diferentes preparos do solo, épocas e posição avaliados.

\begin{tabular}{lcccc}
\hline Causas de variação & DS $\left(\mathbf{M g ~ m}^{-3}\right)$ & DMG $(\mathbf{m m})$ & DMP $(\mathbf{m m})$ & MOS (\%) \\
\hline & \multicolumn{4}{c}{ Camada de 0,00-0,05 m } \\
\hline Manejo & $4,46^{*}$ & $2,04^{\text {ns }}$ & $2,41^{\text {ns }}$ & $0,36^{\text {ns }}$ \\
Época & $27,11^{*}$ & $6,78^{*}$ & $7,24^{*}$ & $0,93^{\text {ns }}$ \\
Manejo*Época & $3,21^{*}$ & $2,13^{\text {ns }}$ & $2,32^{\text {ns }}$ & $1,20^{\text {ns }}$ \\
CV (\%) & 6,8 & 21,8 & 11,2 & 24,6 \\
\hline \multicolumn{5}{c}{ Camada de 0,00-0,05 m } \\
\hline Manejo & $3,59^{*}$ & $1,07^{\text {ns }}$ & $1,32^{\text {ns }}$ & $2,27^{\text {ns }}$ \\
Posição & $4,57^{*}$ & $5,49^{*}$ & $6,85^{*}$ & $10,07^{*}$ \\
Manejo*Posição & $2,34^{\text {ns }}$ & $1,34^{\text {ns }}$ & $1,69^{\text {ns }}$ & $0,95^{\text {ns }}$ \\
CV (\%) & 8,5 & 32,2 & 15,9 & 15,1 \\
\hline
\end{tabular}

* significativo a 0,$05 ; \mathrm{ns}=$ não significativo

Tabela 2. Densidade do solo (Ds), diâmetro médio geométrico (DMG), diâmetro médio ponderado (DMP) e teor de matéria orgânica do solo (MOS\%) dos agregados menores que $8 \mathrm{~mm}$ na camada $0,00-0,05 \mathrm{~m}$, nas diferentes épocas e preparos do solo avaliados.

\begin{tabular}{|c|c|c|c|c|c|}
\hline \multirow[b]{2}{*}{ Época } & \multicolumn{4}{|c|}{ Manejo } & \multirow[b]{2}{*}{ Média } \\
\hline & PD & Esc & EG & ER & \\
\hline \multicolumn{6}{|c|}{ Ds $\left(\mathrm{Mg} \mathrm{m}^{-3}\right)$} \\
\hline 1 & $1,52 \mathrm{Aa}$ & $1,48 \mathrm{Aa}$ & $1,43 \mathrm{Aa}$ & $1,45 \mathrm{Aa}$ & 1,47 \\
\hline 2 & $1,50 \mathrm{Aa}$ & 1,29ABab & 1,26Aab & $1,12 \mathrm{Bb}$ & 1,29 \\
\hline 3 & $1,50 \mathrm{Aa}$ & $1,19 \mathrm{Ba}$ & 1,29ABa & $1,15 \mathrm{Ba}$ & 1,20 \\
\hline Média & 1,51 & 1,32 & 1,33 & 1,24 & \\
\hline \multicolumn{6}{|c|}{ DMG (mm) } \\
\hline 1 & 2,2 & 2,6 & 2,5 & 2,5 & $2,4 a$ \\
\hline 2 & 2,6 & 1,6 & 1,3 & 1,7 & $1,8 b$ \\
\hline 3 & 2,2 & 2,1 & 1,7 & 2,0 & $2,0 \mathrm{ab}$ \\
\hline Média & $2,3 \mathrm{a}$ & $2,1 \mathrm{a}$ & $1,8 \mathrm{a}$ & $2,1 \mathrm{a}$ & \\
\hline \multicolumn{6}{|c|}{ DMP (mm) } \\
\hline 1 & 3,7 & 4,0 & 4,0 & 4,2 & $4,2 \mathrm{a}$ \\
\hline 2 & 4,2 & 3,3 & 2,8 & 3,2 & $3,4 \mathrm{~b}$ \\
\hline 3 & 3,8 & 3,7 & 3,4 & 3,6 & $3,6 a b$ \\
\hline Média & $4,0 \mathrm{a}$ & $3,7 \mathrm{a}$ & $3,4 \mathrm{a}$ & $3,7 \mathrm{a}$ & \\
\hline \multicolumn{6}{|c|}{$\operatorname{MOS}(\%)$} \\
\hline 1 & 2,0 & 2,0 & 1,8 & 1,8 & $1,9 \mathrm{a}$ \\
\hline 2 & 2,0 & 2,0 & 1,8 & 2,3 & $2,1 \mathrm{a}$ \\
\hline 3 & 2,3 & 1,7 & 2,0 & 2,0 & $2,2 \mathrm{a}$ \\
\hline Média & $2,1 \mathrm{a}$ & $1,9 \mathrm{a}$ & $1,9 \mathrm{a}$ & $2,1 \mathrm{a}$ & \\
\hline
\end{tabular}

Médias seguidas de letras iguais, maiúscula na coluna e minúscula na linha, não diferem estatisticamente entre si pelo teste de Tukey a $5 \%$. PD $=$ plantio direto; Esc = escarificado; $\mathrm{EG}=$ escarificado + grade niveladora; $\mathrm{ER}=$ enxada rotativa. 
O maior índice de agregação estimado pelo diâmetro médio geométrico (DMG) e diâmetro médio ponderado (DMP) foi verificado no plantio direto. Porém, essa diferença não foi estatisticamente significativa, quando comparado aos demais sistemas de manejo (Tabela 1 e 2). Para Fageria (2012) e Franchini et al. (2012), a redução ou eliminação do revolvimento do solo associado ao frequente aporte de resíduos vegetais ocorrentes após a adoção do plantio direto, promovem o aumento do teor de matéria orgânica e protegem o solo contra a ação desagregadora da chuva, refletindo em aumento do DMG e DMP. Carpenedo \& Mielniczuk (1990) encontraram agregados maiores e menos densos com a adoção do plantio direto em relação aos sistemas de manejo que promovem o revolvimento do solo. A presença desses agregados grandes pode refletir na resistência do solo à erosão, além de expressar na qualidade dos agregados (Castro Filho et al., 1998).

A degradação temporal da estrutura revela que houve redução do DMG e DMP após alguns meses do preparo, nas épocas 2 e 3 (Tabela 2). Nesse sentido, o maior efeito sobre a agregação foi verificado nos tratamentos escarificado, escarificado seguido de gradagem e enxada rotativa. Entretanto, também apresentaram a recuperação mais rápida da estrutura, provavelmente em decorrência da incorporação dos resíduos vegetais durante o revolvimento (Fuentes et al., 2009), que favorecem a mineralização do carbono (Kumaria et al., 2011). A influência benéfica da matéria orgânica sobre a agregação do solo é um processo dinâmico e está associada à intensificação da atividade microbiana (Riley et al., 2008) em função da presença de fungos e bactérias presentes no solo (Tisdall \& Oades, 1982; Tang et al., 2011), que promovem a ligação entre polímeros orgânicos e a superfície inorgânica por meio de cátions polivalentes (Edwards \& Bremmer, 1967).

Como o principal agente de estabilização dos agregados do solo é a matéria orgânica (Lal, 2009), esperava-se a ocorrência de correlação entre o teor de matéria orgânica e a agregação. Porém os resultados deste trabalho não confirmaram essa expectativa, pois o DMG e DMP não apresentaram correlação significativa com a matéria orgânica (Tabela 3), semelhante ao obtido por Riley et al. (2008), o qual explica que a pequena variação no conteúdo de matéria orgânica antes e após o preparo do solo pode não ter sido suficiente para afetar a estabilidade de agregados. As plantações de eucalipto podem preservar os estoques de matéria orgânica, mantendo o estado de agregação do solo (Zinn et al., 2011), devido ao sistema radicular das árvores que, durante o seu crescimento, exercem pressão aproximando as partículas do solo, liberam exsudatos e promovem ciclos de umedecimento e secagem, predispondo à formação de agregados e à estabilização da estrutura do solo (Lal, 2009), mesmo em árvores que se encontram em estágio inicial de crescimento (Gonçalves, 2002). Entretanto, os diferentes preparos do solo avaliados não foram capazes de aumentar o teor de carbono orgânico, não influenciado no estado de agregação.

Tabela 3. Coeficientes de correlação linear de Pearson e significância entre as variáveis testadas nas diferentes épocas de avaliação na linha de plantio.

\begin{tabular}{|c|c|c|c|c|c|}
\hline & & MOS & Ag. $<0,25$ & Ag. 8,0 - 4,76 & DMP \\
\hline \multirow{4}{*}{ Época 1} & DMG & $0,04^{\mathrm{ns}}$ & $-0,72 * *$ & $0,92 * * *$ & $0,97 * * *$ \\
\hline & DMP & $-0,02^{\mathrm{ns}}$ & $-0,70^{* *}$ & $0,97 * * *$ & - \\
\hline & Ag. 8,0 - 4,76 & $0,01^{\mathrm{ns}}$ & $-0,58^{*}$ & - & - \\
\hline & Ag. $<0,25$ & $0,19^{\text {ns }}$ & - & - & - \\
\hline \multirow{4}{*}{ Época 2} & DMG & $-0,001^{\mathrm{ns}}$ & $-0,64^{*}$ & $0,54^{*}$ & $0,97 * * *$ \\
\hline & DMP & $-0,003^{\mathrm{ns}}$ & $-0,63 *$ & $0,57^{*}$ & - \\
\hline & Ag. 8,0 - 4,76 & $0,05^{\mathrm{ns}}$ & $-0,41^{\mathrm{ns}}$ & - & - \\
\hline & Ag. $<0,25$ & $0,34^{\mathrm{ns}}$ & - & - & - \\
\hline \multirow{4}{*}{ Época 3} & DMG & $-0,26^{\mathrm{ns}}$ & $-0,87 * * *$ & $0,89 * * *$ & $0,95 * * *$ \\
\hline & DMP & $-0,30^{\mathrm{ns}}$ & $-0,77 * * *$ & $0,98 * * *$ & - \\
\hline & Ag. 8,0 - 4,76 & $-0,28^{\mathrm{ns}}$ & $-0,66^{*}$ & - & - \\
\hline & Ag. $<0,25$ & $-0,02^{\mathrm{ns}}$ & - & - & - \\
\hline
\end{tabular}

$\mathrm{DMG}=$ diâmetro médio geométrico; $\mathrm{DMP}=$ diâmetro médio ponderado; $\mathrm{MOS}=$ matéria orgânica do solo; $\mathrm{Ag} .<0,25$ : classe de tamanho de agregados $<0,25$; Ag. 8,0 - 4,76: classe de tamanho de agregados de $8,0-4,76 . *$ significativo a 0,$05 ; * *$ significativo a 0,$01 ; * * *$ significativo a 0,$001 ;$ ns $=$ não significativo. 
Os diferentes preparos do solo empregados na implantação do povoamento de Eucaliptus grandis e posição de coleta não apresentaram interação significativa para a densidade nas épocas 2 e 3 (Tabela 4). Na época 2 , a maior densidade foi verificada no plantio direto em comparação aos demais preparos do solo, com significativa redução na linha e entrelinha de plantio. Na época 3, essa diferença de densidade entre os preparos do solo não foi visualizada, demonstrando a recuperação da estrutura resultante do cultivo do eucalipto após transcorridos 12 meses de plantio. Essa mesma tendência foi encontrada para o DMG e o DMP, os quais não apresentaram diferença significativa entre a linha e entrelinha de plantio nos diferentes preparos, nas épocas 2 e 3 . Esses resultados confirmam que o revolvimento mínimo do solo, com preparos localizados na linha de plantio, não provocam degradação da estrutura do solo e favorecem a sustentabilidade da produção por períodos mais longos.

Tabela 4. Densidade do Solo (Ds), diâmetro médio geométrico (DMG), diâmetro médio ponderado (DMP) e teor de matéria orgânica do solo (MOS\%) dos agregados menores que $8 \mathrm{~mm}$ da camada $0,00-0,05 \mathrm{~m}$ aos 3 meses (época 2) e aos 12 meses após o preparo do solo (época 3), determinados na linha e entrelinha do Argissolo.

\begin{tabular}{|c|c|c|c|c|c|}
\hline \multirow{2}{*}{ Local } & \multicolumn{4}{|c|}{ Manejo } & \multirow{2}{*}{ Média } \\
\hline & PD & Esc & EG & ER & \\
\hline \multicolumn{6}{|c|}{ Ds $\left(\mathrm{Mg} \mathrm{m}^{-3}\right)$} \\
\hline $\mathbf{E}$ & 1,40 & 1,37 & 1,45 & 1,35 & $1,39 \mathrm{a}$ \\
\hline $\mathbf{L}$ & 1,50 & 1,29 & 1,26 & 1,12 & $1,29 b$ \\
\hline Média & $1,45 \mathrm{a}$ & $1,33 \mathrm{ab}$ & $1,36 \mathrm{ab}$ & $1,24 b$ & \\
\hline \multicolumn{6}{|c|}{ DMG (mm) Época 2} \\
\hline $\mathbf{E}$ & 2,6 & 2,4 & 2,5 & 2,4 & $2,5 \mathrm{a}$ \\
\hline $\mathbf{L}$ & 2,6 & 1,6 & 1,3 & 1,7 & $1,8 \mathrm{a}$ \\
\hline Média & $2,6 a$ & $2,0 \mathrm{a}$ & $1,9 \mathrm{a}$ & $2,0 \mathrm{a}$ & \\
\hline \multicolumn{6}{|c|}{ MOS (\%) Época 2} \\
\hline $\mathbf{E}$ & 2,8 & 2,6 & 2,1 & 2,5 & $2,5 \mathrm{a}$ \\
\hline $\mathbf{L}$ & 2,0 & 2,0 & 1,8 & 2,3 & $2,1 b$ \\
\hline Média & $2,4 \mathrm{a}$ & $2,3 \mathrm{a}$ & $2,0 \mathrm{~b}$ & $2,4 \mathrm{a}$ & \\
\hline \multicolumn{6}{|c|}{ Ds $\left(\mathrm{Mg} \mathrm{m}^{-3}\right)$} \\
\hline $\mathbf{E}$ & 1,45 & 1,50 & 1,43 & 1,49 & $1,47 \mathrm{a}$ \\
\hline $\mathbf{L}$ & 1,43 & 1,20 & 1,30 & 1,15 & $1,20 \mathrm{~b}$ \\
\hline Média & $1,44 \mathrm{a}$ & $1,35 \mathrm{a}$ & $1,36 \mathrm{a}$ & $1,32 \mathrm{a}$ & \\
\hline \multicolumn{6}{|c|}{ DMG (mm) Época 3} \\
\hline $\mathbf{E}$ & 2,1 & 2,2 & 2,1 & 2,8 & $2,3 \mathrm{a}$ \\
\hline $\mathbf{L}$ & 2,2 & 2,1 & 1,8 & 1,9 & $2,0 \mathrm{a}$ \\
\hline Média & $2,1 \mathrm{a}$ & $2,1 \mathrm{a}$ & $1,9 \mathrm{a}$ & $2,3 \mathrm{a}$ & \\
\hline \multicolumn{6}{|c|}{ MOS (\%) Época 3} \\
\hline $\mathbf{E}$ & 2,3 & 2,0 & 2,4 & 1,7 & $2,1 \mathrm{a}$ \\
\hline $\mathbf{L}$ & 2,3 & 1,7 & 2,7 & 2,0 & $2,2 \mathrm{a}$ \\
\hline Média & $2,3 \mathrm{a}$ & $1,9 \mathrm{a}$ & $2,6 a$ & $1,9 \mathrm{a}$ & \\
\hline
\end{tabular}

Médias seguidas de letras iguais, na coluna e na linha, não diferem estatisticamente entre si pelo teste de Tukey a 5\%. PD = plantio direto; Esc = escarificado; $\mathrm{EG}=$ escarificado + grade niveladora; $\mathrm{ER}=$ enxada rotativa. 
A porcentagem de agregados nas classes de maior diâmetro $(8,00-4,76 \mathrm{~mm})$ foi reduzida nos preparos que revolveram o solo, com consequente aumento na classe de menor diâmetro $(<0,25 \mathrm{~mm})$ em relação ao plantio direto, quando comparam-se a linha e entrelinha de plantio (Figura 2). Os tratamentos escarificado e escarificado seguido de gradagem foram os mais afetados, com um aumento de $50 \%$ para a classe de menor diâmetro, fato relacionado ao preparo do solo que provoca a quebra dos agregados maiores, com favorecimento a mineralização da matéria orgânica, principalmente no tratamento escarificado seguido de gradagem, após três meses do manejo (época 2) na linha de plantio. O não revolvimento e o acúmulo e incorporação de material orgânico sobre a superfície do solo no plantio direto resultaram em efeito positivo sobre a estabilidade dos agregados, concordando com o descrito por Carpenedo \& Mielniczuk (1990), Fuentes et al. (2009), Fageria (2012) e Franchini et al. (2012).
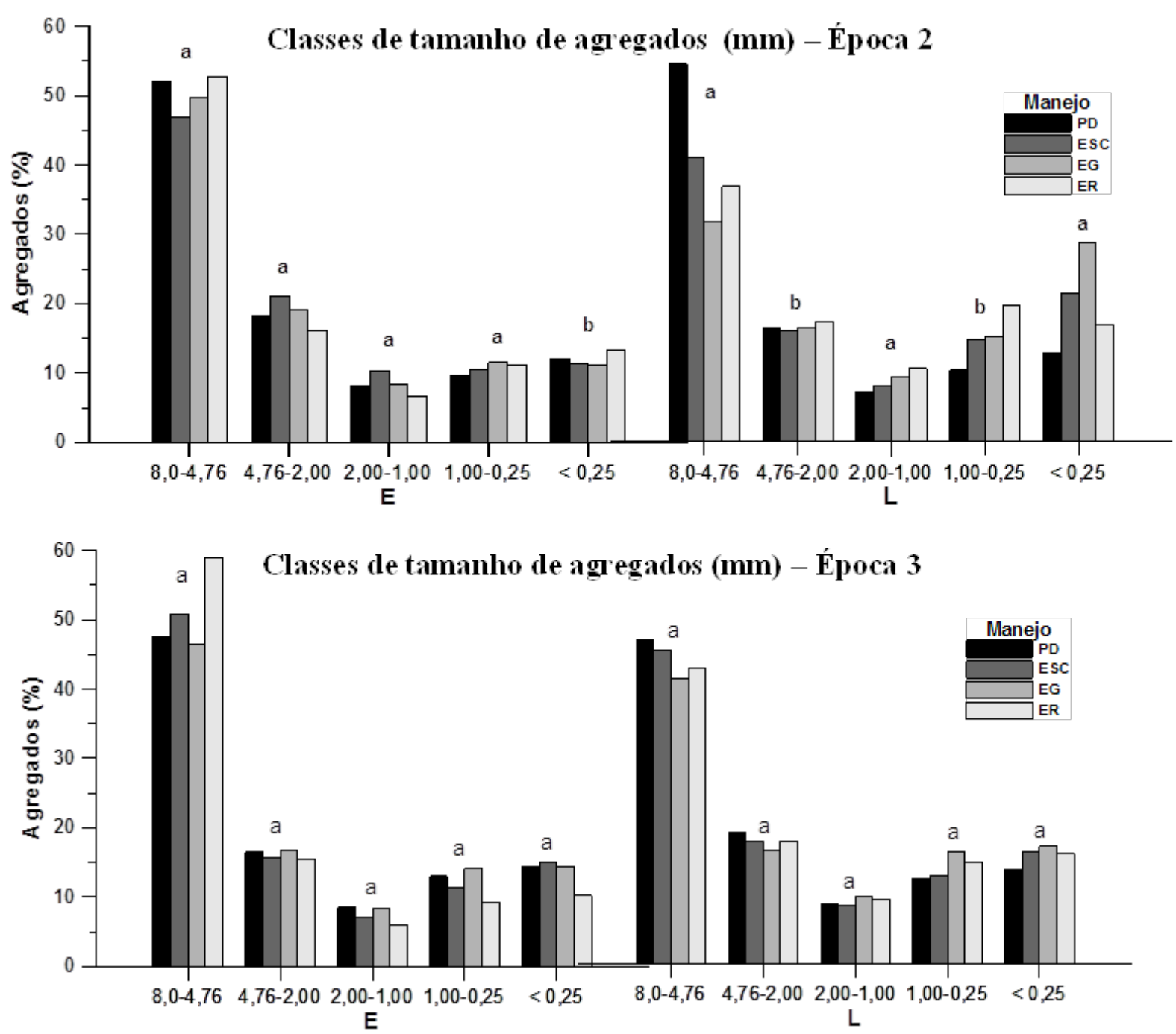

Figura 2. Distribuição do tamanho de agregados estáveis em água (menor que $8 \mathrm{~mm}$ ) em Argissolo determinados na entrelinha (E) e linha (L) de plantio para cada sistema de manejo na época 2 e época 3. $\mathrm{PD}=$ plantio direto; Esc = escarificado; $\mathrm{EG}=$ escarificado + grade niveladora; $\mathrm{ER}=$ enxada rotativa. Valores seguidos de mesma letra para cada classe de agregado menor que $8 \mathrm{~mm}$ na entrelinha e linha de plantio, não diferem significativamente pelo teste de Tukey a $5 \%$ de probabilidade de erro. 
Doze meses após o preparo, verificou-se a recuperação espacial da agregação do solo, evidenciada pelo aumento da percentagem de agregados nas classes de maior diâmetro, o que pode ser atribuído à reconsolidação natural do solo e à ação mecânica das raízes do eucalipto. Gonçalves (2002), Bernoux et al. (2009), Beldini et al. (2010), Zinn et al. (2011) e Fonte et al. (2012) relatam que as espécies florestais possuem grande potencial na formação e estabilização de agregados, devido ao sistema radicular denso e aporte elevado e regular de resíduos orgânicos ao solo, além de protegerem a superfície quanto ao efeito de desagregação da chuva.

Os parâmetros dendrométricos do E. grandis aos 12 meses de idade foram afetados de forma significativa pelo sistema de preparo utilizado para o estabelecimento da floresta (Tabela 5). De um modo geral, houve tendência de aumento da sobrevivência e do diâmetro à altura do peito (DAP), com reflexos na área basal e no volume, à medida que se intensificou o preparo do solo, com superioridade de DAP de $42 \%$ no tratamento enxada rotativa em relação ao planto direto após 12 meses de plantio. Esta tendência evidencia a importância do revolvimento do solo no início do desenvolvimento das árvores, pois o crescimento inicial do eucalipto é favorecido pela intensidade do preparo do solo entre o primeiro e segundo ano, podendo se estender até o final do ciclo (Gonçalves, 2002). Finger et al. (1996) encontraram um crescimento em DAP superior a 50\% em solo subsolado em relação ao não subsolado no povoamento com mais de 24 meses de idade.

Tabela 5. Valores médios de diâmetro à altura do peito (DAP), altura, área basal, volume e sobrevivência do E. grandis aos 12 meses de idade, nos diferentes manejos.

\begin{tabular}{cccccc}
\hline Manejo & $\begin{array}{c}\text { DAP } \\
(\mathbf{c m})\end{array}$ & $\begin{array}{c}\text { Altura } \\
(\mathbf{m})\end{array}$ & $\begin{array}{c}\text { Área } \\
\text { basal } \\
\left(\mathbf{m}^{\mathbf{2}} \mathbf{h a}^{-\mathbf{1}}\right)\end{array}$ & $\begin{array}{c}\text { Volume } \\
\left(\mathbf{m}^{\mathbf{3}} \mathbf{h a} \mathbf{- 1}\right)\end{array}$ & $\begin{array}{c}\text { Sobrevivência } \\
\mathbf{\%}\end{array}$ \\
\hline PD & $1,57 \mathrm{a}$ & $2,40 \mathrm{a}$ & $0,31 \mathrm{a}$ & $1,07 \mathrm{a}$ & $59 \mathrm{a}$ \\
Esc & $1,95 \mathrm{ab}$ & $2,65 \mathrm{a}$ & $0,59 \mathrm{~b}$ & $1,99 \mathrm{ab}$ & $82 \mathrm{ab}$ \\
EG & $2,06 \mathrm{ab}$ & $2,80 \mathrm{a}$ & $0,65 \mathrm{~b}$ & $2,23 \mathrm{ab}$ & $87 \mathrm{ab}$ \\
ER & $2,69 \mathrm{~b}$ & $3,58 \mathrm{~b}$ & $1,09 \mathrm{c}$ & $4,76 \mathrm{~b}$ & $91 \mathrm{c}$ \\
\hline
\end{tabular}

Médias seguidas de letras iguais na coluna não diferem estatisticamente entre si pelo teste de Tukey a 5\%. PD = plantio direto; Esc = escarificação; $\mathrm{EG}=$ escarificação e gradagem; $\mathrm{ER}$ = enxada rotativa.

O plantio direto resultou em $59 \%$ de sobrevivência das plantas. Esse baixo índice pode estar relacionado à maior dificuldade de estabelecimento das raízes no período inicial do desenvolvimento das plantas, devido ao não revolvimento do solo e a maior competição com plantas daninhas. Porém, o aumento da intensidade de preparo resultou em maior sobrevivência das mudas, onde o solo que recebeu preparo do solo com enxada rotativa foi o que resultou em maior número de plantas (91\%). Finger et al. (1996) encontraram 97,6\% de sobrevivência em solo subsolado e $92,9 \%$ em solo que não recebeu subsolagem.

\section{Conclusões}

Os preparos do solo com escarificação, escarificação seguida de gradagem e com enxada rotativa resultaram em menor estabilidade estrutural de agregados estáveis em água, com redução da porcentagem de agregados na classe de maior diâmetro (4,76-8,00 mm).

Os efeitos negativos do preparo do solo sobre o Argissolo de textura franco arenosa foram reduzidos após 12 meses, demonstrando sua capacidade em restabelecer a estrutura e a agregação.

Os diferentes preparos do solo avaliados não foram capazes de aumentar o teor de carbono orgânico, no espaço de 12 meses, não influenciado no estado de agregação.

A intensificação do preparo do solo favoreceu a sobrevivência e o crescimento inicial do eucalipto, com porcentagem de sobrevivência e diâmetro à altura do peito superior em $35 \%$ e $42 \%$ no preparo com enxada rotativa em relação ao plantio direto, respectivamente.

\section{Referências}

BAUMGARTL, T.; HORN, R. Effect of aggregate stability on soil compaction. Soil Tillage Research, Amsterdam, v. 19, n. 2-3, p. 203-213, 1991. DOI: 10.1016/j.bbr.2011.03.031.

BELDINI, T. P.; MCNABB, K. L.; LOCKABY, B. G.; SANCHEZ, F. G.; NAVEGANTES-CÂNCIO, O. The effect of Amazonian Eucalyptus plantations on soil aggregates and organic matter density fractions. Soil Use and Management, Amsterdam, v. 26, n. 1, p. 53-60, 2010. DOI: 10.1111/j.1475-2743.2009.00248.x.

BERNOUX, M.; CERRI, C. C.; CERRI, C. E. P.; SIQUEIRANETO, M.; METAY, A.; PERRIN, A.-S.; SCOPEL, E.; RAZAMFIMBELO, T.; BLAVET, D.; PICCOLO, M. de C.; MILNE, E. Cropping systems, carbon sequestration and erosion in Brazil: A review. In: LICHTFOUSE, E.; NAVERRETE, M.; DEBAEKE, P.; SOUCHERE, V.; ALBEROLA, C. (Ed.). Sustainable agriculture. Berlin: Springer-Verlag, 2009. p. 75-85.

CARPENEDO, V.; MIELNICZUK, J. Estado de agregação e qualidade de agregados de Latossolos Roxos, submetidos a diferentes sistemas de manejo. Revista Brasileira de Ciência do Solo, Campinas, v. 14, n. 1, p. 99-105, 1990. 
CASTRO FILHO, C.; MUZILLI, O.; PADANOSCHI, A. L. Estabilidade dos agregados e sua relação com o teor de carbono orgânico num Latossolo Roxo distrófico, em função de sistemas de plantio, rotações de culturas e métodos de preparo das amostras. Revista Brasileira de Ciência do Solo. Viçosa, MG, v. 22, n. 3, p. 527-538, 1998.

CLAESSEN, M. E. C. (Org.). Manual de métodos de análise de solo. 2. ed. rev. atual. Rio de Janeiro: EMBRAPA-CNPS, 1997. 212 p.

EDWARDS, A. P.; BREMNER, J. M. Microaggregates in soils. Journal of Soil Science. Victoria, v. 18, n. 1, p. 64-73, 1967. DOI: 10.1111/j.1365-2389.1967.tb01488.x.

FAGERIA, N. K. Role of soil organic matter in maintaining sustainability of cropping systems. Communications in Soil Science and Plant Analysis, Philadelphia, v. 43, n. 16, p. 2063-2113. 2012. DOI: 10.1080/00103624.2012.697234.

FINGER, C. A. G.; SCHUMACHER, M. V.; SCHNEIDER, P. R.; HOPPE, J. H. Influência da camada de impedimento no solo sobre o crescimento de Eucalyptus grandis (Hill) ex Maiden. Ciência Florestal, Santa Maria, RS, v. 6, n. 1, p. 137-145, 1996.

FONTE, S. J.; QUINTERO, D. C.; VELÁSQUEZ, E.; LAVELLE, P. Interactive effects of plants and earthworms on the physical stabilization of soil organic matter in aggregates. Plant and Soil, The Hague, v. 359, n. 1-2, p. 205-214, 2012. DOI: 10.1007/s11104-012-1199-2.

FRANCHINI, J. C.; DEBIASI, H.; BALBINOT JUNIOR, A. A. B.; TONON, B. C.; FARIAS, J. R. B.; DE OLIVEIRA, M. C. N.; TORRES, E. Evolution of crop yields in different tillage and cropping systems over two decades in southern Brazil. Field Crops Research, Amsterdam, v. 137, n. 20, p. 178-185, 2012. DOI: 10.1016/j.fcr.2012.09.003.

FUENTES, M.; GOVAERTS, B.; DE LEÓN, F.; HIDALGO, C.; DENDOOVEN, L.; SAYRE, K. D.; ETCHEVERS, J. Fourteen years of applying zero and conventional tillage, crop rotation and residue management systems and its effect on physical and chemical soil quality. European Journal of Agronomy, Amsterdam, v. 30, n. 3, p. 228-237, 2009. DOI: 10.1016/j.eja.2008.10.005.

GONÇALVES, J. L. M. Principais solos usados para plantações florestais. In: GONÇALVES, J. L. M.; STAPE, J. L. Conservação e cultivo de solos para plantações florestais. Piracicaba: IPEF, 2002, p.1-46.

KEMPER, W. D.; CHEPIL, W. S. Size distribution of aggregates. In: BLACK, C. A.; EVANS, D. D.; WHITE, J. L. (Ed.). Methods of soil analysis: part 1. Madison: American Society of Agronomy, 1965. p. 499-509.

KUMARIA, M.; CHAKRABORTYA, D.; GATHALAB, M. K.; PATHAKC, H.; DWIVEDID, B. S.; GARGA, T. R. R. N.; SINGHA, R.; LADHA, J. K. Soil aggregation and associated organic carbon fractions as affected by tillage in a rice-wheat rotation in North India. Soil Science Society of America Journal, Madison, v. 75, n. 2, p. 560-567. 2011. DOI: 10.2136/sssaj2010.0185.

LAL, R. Challenges and opportunities in soil organic matter research. European Journal of Soil Science, Amsterdam, v. 60, n. 2, p. 158-169, 2009. DOI: 10.1111/j.1365-2389.2008.01114.x.

LETEY, J. The study of soil structure: science and art. Australian Journal of Soil Research. Collingwood, v. 29, n. 6, p. 699-707, 1991. DOI: 10.1071/SR9910699.

MOREnO, J. A. Clima do Rio Grande do Sul. Porto Alegre: Secretaria da Agricultura, 1961. $42 \mathrm{p}$.

Pesq. flor. bras., Colombo, v. 34, n. 78, p. 149-158, abr.jun. 2014
PIKUL JUNIOR, J. L., CHILOM, G., RICE, J., EYNARD, A., SCHUMACHER, T. E., NICHOLS, K., JOHNSON, J. M. F., WRIGHT, S., CAESAR, T., ELLSBURY, M. Organic matter and water stability of field aggregates affected by Tillage in South Dakota. Soil Science Society of America Journal. Madison, v. 73, n. 1, p. 197-206, 2009. DOI: 10.2136/sssaj2007.0184.

REICHERT, J. M.; SUZUKI, L. E. A. S.; REINERT, D. J.; HORN, R.; HAKANSSON, I. Reference bulk density and critical degreeof compactness for no-till crop production in subtropical highly weathered soils. Soil \& Tillage Research, Amsterdam, v. 102, n. 2, p. 242-254, 2009. DOI: 10.1016/j.still.2008.07.002.

REICHERT, J. M.; ALBUQUERQUE, J. A.; GUBIANI, P. I.; KAISER, D. R.; MINELLA, J. P. G.; REINERT, D. J. Hidrologia do solo, disponibilidade de água às plantas e zoneamento agroclimático. In: KLAUBERG FILHO, O.; MAFRA, A. L.; GATIBONI, L. C. (Ed.). Tópicos em ciência do solo. Viçosa, MG: SBCS, 2011. v. 7. p. 1-54.

RILEY, H.; POMMERESCHE, R.; ELTUN, R.; HANSEN, S.; KORSAETH, A. Soil structure, organic matter and earthworm activity in a comparison of cropping systems with contrasting tillage, rotations, fertilizer levels and manure use. Agriculture, Ecosystems \& Environment, Amsterdam, v. 124, n. 3-4, p. 275-284, 2008. DOI: 10.1016/j.agee.2007.11.002.

SAHA, S. K.; NAIR, P. K. R.; NAIR, V. N.; KUMAR, B. M. Carbon storage in relation to soil size-fractions under tropical tree-based landuse systems. Plant and Soil, The Hague, v. 328, n. 1-2, p. 433-446, 2010. DOI:10.1007/s11104-009-0123-X.

SANTOS, H. G. dos; JACOMINE, P. K. T.; ANJOS, L. H. C. dos; OLIVEIRA, V. A. de; OLIVEIRA, J. B. de; COELHO, M. R.; LUMBRERAS, J. F.; CUNHA, T. J. F. (Ed.). Sistema brasileiro de classificação de solos. 2. ed. Rio de Janeiro: Embrapa Solos, 2006.306 p.

TANG, J.; MO, Y.; ZHANG, J.; ZHANG, R. Influence of biological aggregating agents associated with microbial population on soil aggregate stability. Applied Soil Ecology, Amsterdam, v. 47, n. 3, p. 153-159, 2011. DOI: 10.1016/j.apsoil.2011.01.001.

TEDESCO, M. J.; GIANELLO, C.; BISSANI, C. A.; BOHNEN, H.; VOLKWEIIS, S. J. Análise de solo, plantas e outros materiais. 2. ed. Porto Alegre: Ed da UFRGS, 1995. 174 p. (Boletim técnico, 5).

TISDALL, J. M.; OADES, J. M. Organic matter and water-stable aggregates in soils. Journal of Soil Science, Victoria, v. 33, n. 2, p. 141-163, 1982. DOI: 10.1111/j.1365-2389.1982.tb01755.x.

YANG, Y.; GUO, J.; CHEN, G.; YIN, Y.; GAO, R.; LIN, C. Effects of forest conversion on soil labile organic carbon fractions and aggregate stability in subtropical China. Plant and Soil, The Hague, v. 323, n. 1-2, p. 153-162, 2009. DOI: 10.1007/s11104-009-9921-4.

YODER, R. E. A direct method of aggregate analysis of soils and a study of the physical nature of erosion losses. Journal of the American Society of Agriculture, Madison, v. 28, n. 5, p. 337-351, 1936.

ZINN, Y. L.; LAL, R.; BIGHAM, J. M.; RESCK, D. V. S. Edaphic controls on soil organic carbon retention in the Brazilian Cerrado: soil structure. Soil Science Society of America Journal. Madison, v. 71, n. 4, p. 1215-1224, 2007. DOI:10.2136/sssaj2006.0015.

ZINN, Y. L.; LAL, R.; RESCK, D. V. S. Eucalypt plantation effects on organic carbon and aggregation of three different-textured soils in Brazil. Soil Research, Collingwood, v. 49, n. 7, p. 614-624, 2011. DOI: $10.1071 /$ SR11264. 